\title{
Transverse Wake Effects in an Optical Waveguide IFEL Accelerator
}

\author{
J. Sandweiss* \\ National Synchrotron Light Source \\ Center for Accelerator Physics \\ Brookhaven National Laboratory \\ Upton, New York 11973
}

\section{NATIONAL SYNCHROTRON LIGHT SOURCE}

* Visitor from Yale University, New Haven, Comnerticut

$$
\begin{aligned}
& \text { BROOKHAVEN NA: ONAL LAB:UAY :RV }
\end{aligned}
$$

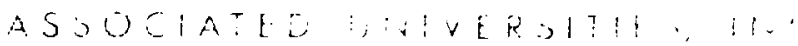


TRANSVERSE WAKE EFFECTS in an OPTICAL WAVEGUIDE

IFEL ACCELERATOR

J. Sandweiss

August 13,1987

BNL -40310

DE88 004591

\section{Introduction}

A recent prorosal [1] describes a project to build a short demonstration stage IFEL accelerator using as input beam the output of the Accelerator Test Facility high brightness linac (ATF) currently under construction at BNL. A second stage of the proposal involves the design and construction of a $1 \mathrm{GeV}$ IFEL accelerator.

Both IFEL accelerators could be built using standard focussing optics as discussed in the proposal. While this is fairly natural in the short $(60 \mathrm{~cm})$ demonstration accelerator, it requires multiple refocussing for the $1 \mathrm{GeV}$ machine. It would therefore be valuable if an optical waveguide could be used to contain the laser beam over the full length of the accelerator. Such waveguides, with very low losses, have been described previously [2].

However, it is clear that if the use of a waveguide is to compete with the direct focussing method, the aper'ure of the waveguide must be comparable to the laser beam waist for a Rayleigh length of $\approx 30 \mathrm{~cm}$. In other words, the waveguide aperture must be at most a few rum. A natural concern for such close fitting structures is the effect of the wake fields produced in the waveguide by the beam. In particular, the wake rields produced by the front of the beam pulse may lead to transverse oscillations of the rear of the beam pulse.

In the remaincier of this paper Wilson's two particle model [3] of this headtail effect is used to estimate the magnitude of the emittance growth in an optical waveguide IFEL accelerator.

\section{Choice of Waveguide Dimensions}

In this section we calculate a minimum dimension such that the flatness of the laser electric field across the transverse dimension of the beam is as good as it is in the direct focussing case described in the propssal. The wriveguide dimension can be larger than this minimum but so doing will use the laser porrer less efficiently. It may, of course, be necessary to do so for other reasons.

In the Gaussian beam with Rayleigh length $R$ the transverse electric field at waist, $E_{l}$, may be written:

$$
E_{l} \sim e^{r^{2} / w_{0}^{2}}
$$

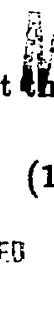


where

$$
w_{0}^{2}=\frac{\lambda R}{\pi}
$$

For $\lambda=10 \mu \mathrm{m}$ and $R=30 \mathrm{~cm}, w_{0}=0.0977 \mathrm{~cm}$ and the r.m.s. radius for $E_{l}$ is $.69 \mathrm{~mm}$. The total power in the laser beam, $P$, is:

$$
P=K \int_{-\infty}^{+\infty} \int_{-\infty}^{+\infty} E_{l}^{2} d x d y
$$

where $K$ is an appropriate constant.

If we take $E_{l}(0,0)=1$,

$$
P=\frac{K \pi w_{0}^{2}}{2}=\bar{K}\left(1.496 \mathrm{~mm}^{2}\right)
$$

To a very good approximation, the electric field in the waveguide is given by

$$
E_{l}=\frac{\cos \pi x}{D} \frac{\cos \pi y}{D}
$$

where $\mathrm{D}$ is the half aperture of the (assumed) square guide.

Again take $E_{l}(0,0)=1$, whence the total laser power is:

$$
P=K D^{2}
$$

To choose the minimum $D$ we ask that the central flatness for the two approaches be the same. Now the radius of the ATF beam (assuming a $\beta$ function value of 1 meter) is the quadrature of the basic beam radius $(1 \sigma)$ of $\sim .3 \mathrm{~mm}$ and the oscillation amplitude in the wiggler of $\sim .185 \mathrm{~mm}$ giving a $(1 \sigma)$ beam radius of $.35 \mathrm{~mm}$. The ratio of the field at the edge to the central field for the focussed laser beam is thus:

$$
\frac{E_{l}(r=.35 \mathrm{~mm})}{E_{l}(0)}=e^{\frac{-(.35)^{2}}{2(.00)^{2}}}=.879
$$

We now say that the same decrease should apply in the waveguide case at

$$
x=y=\frac{\tau_{\text {beam }}}{\sqrt{2}}=\frac{.35}{\sqrt{2}}=.2475
$$

so

$$
\cos ^{2}\left(\frac{.2475 \pi}{2 D}\right)=.879
$$

whence $D=1.09 \mathrm{~mm}$. At this $\mathrm{D}$ the total laser power is from (6), $K\left(1.2 \mathrm{~mm}^{2}\right)$, which is less than the total power (eq. 4) for the focussed beam.

This treatment is admittedly approximate. However, the main result that the minimum waveguide aperture will be around $\pm 1 \mathrm{~mm}$ from field flatness requirements is plausible and cannot be too far off. Furthermore this calculation shows that at 
comparable flatness, the waveguide does indeed concentrate the laser beam at least as efficiently as the externally focussed case. Of course, the waveguide has the advaniage of maintaining a constant laser intensity throughout its length.

\section{Two Particle Model for the Transverse Emmittance Growth}

Wilson [3] has developed a simple two particle nodel which describes the effects of the transverse wake fielo's. This model has been compared with more accurate computer models and has been shown to describe the emmittance growth correctly within a factor of the order of 1.5 to 2 .

In the two particle model, we imagine the electrons in the bunch as grouped into two quasi paricles, a head particle and a tail particle, each with charge $\frac{q}{2}$ where $q$ is the total bunch charge. We further assume that the head particle moves at a constant radial displacement of $x_{0}$ as it travels down the guide. Because the head particle is off axis it will create a wake which has transverse deflecting fields which will act upon the tail particle.

Let $W_{d}(\xi)$ be the the transverse force on a tail particle trailing by $\xi$, per unit head charge per unit head displacement per unit tail charge. The subscript $d$ denotes the dipole wake which is the dominant effect in our case. The quadrupole and higher order wakefields vanish on axis.

For simplicity, we consider motion only in one transverse dimension say $x$. The actual motion will, of course, be a superposition of motions in the two transverse planes. The transverse equation of motion of an electron in the tail bunch is:

$$
\frac{d P_{x}}{d t}=e \frac{q}{2} x_{0} W_{d}^{\prime}(\xi)
$$

thus

$$
m \gamma c \dot{\beta}_{x}=e \frac{q}{2} x_{0} W_{d}(\xi)
$$

using $\dot{\beta}_{z}=c \frac{d^{2} x}{d t^{2}}$ leads to

$$
x^{\prime \prime}=\frac{e q x_{0}}{2 m \gamma c^{2}} W_{d}(\xi)
$$

The quantity $m \gamma c^{2}$ is the electron total energy which can he written $e V_{0}$ whence

$$
x^{\prime \prime}=\frac{q x_{0}}{2 V_{0}} W_{d}(\xi)=C
$$

From the point of view of the $x$ motion the right hand side of (8) is constant and the equation can be trivially integrated.

$$
\begin{gathered}
x^{\prime}=C s+\theta_{0} \\
x=\frac{C s^{2}}{2}+\theta_{0} s+a
\end{gathered}
$$


Where $s$ represents distance along the beam measured from the beginning of the waveguide and $\theta_{0}$ and $a$ are initial constants.

In this treatment we have neglected focussing in the transverse motion but it is easy to modify the theory to include it [3]. The wakefield effects are most serious in the absence of focussing so our results will be upper limits. In short, the emmittance growth with focussing will be smaller by a factor equal to the number of betatron oscillations $(x 2 \pi)$ which can occur within the waveguide length.

\section{Calculation of Wakeflelds}

The optical waveguide described in reference [2] is approximately a rectangular cross section metallic waveguide with very thin dielectric coatings on two opposing faces. From the point of view of the wakefield calculation the dielectric coatings can be neglected.

Other guide structures are possible. For example, a purely metallic guide consisting of top and bottom cylindrical reflectors has been suggested [4]. All the waveguide structures have the characteristic of smooth walls and are longitudinally homogeneous. Therefore, a good first approximation for wakefield calculation will be to take the case of a smooth round tube with finite conductivity. This structure also has the advantage that an analytical solution is available[5,6].

In MKS units, the formula for the transverse dipole wake $W_{d}$ is

$$
W_{d}(\xi)=\frac{1}{\pi \epsilon_{0} 3^{3}} \sqrt{\frac{1}{\pi \sigma Z_{0}}} \frac{1}{\xi^{\frac{1}{2}}}
$$

where

$\epsilon_{0}=8.85 \times 10^{-12}$ Farads per Meter

$b=$ radius of the beam tube

$\sigma=$ conductivity of the tube material

$Z_{0}=377$ Ohms per square (free space impedance)

$\xi=$ the distance between head and tail particle

\section{Results}

We now calculate the growth in the $x$ coordinate of a typical tail electron due to wake field effects over the length of the accelerator. We shall consider two accelerators, the short, $60 \mathrm{~cm}$, demonstration stage and the $1 \mathrm{GeV}$ accelerator which we take to be $6 m$ in length. The calculations will be carried out for several different values of the guide radius $b$.

Referring to the properties of the ATF beam [1] we choose

$q=6 \times 10^{9} e=9.6 \times 10^{-10}$ Coulomb

$\xi=1 \mathrm{~mm}$

For the conductivity we use that of copper: 
$\sigma=5.8 \times 10^{7}$ mho per meter

And we make the reasonable assumption that the (constant) displacement of the head particle is

$$
x_{0}=.1 \mathrm{~mm}
$$

For the short accelerator we take a constant $V_{0}$ equal to the average of $75 \mathrm{MeV}$. For the $1 \mathrm{GeV}$ accelerator we take $V_{0}$ to be $450 \mathrm{MeV}$. The results for these conditions are presented in tables I and II.

\section{Table I}

Growth in Transverse Angle and Amblitude due to Wakefleld Effects in the $60 \mathrm{~cm}$ IFEL Demonstration Stage Accelerator

$\begin{array}{lll}b(\mathrm{~mm}) & \Delta \theta(\mathrm{rad}) & \Delta x(\text { meters }) \\ 1 & 1.67 \times 10^{-6} & 5 \times 10^{-7} \\ 2 & 2.08 \times 10^{-7} & 6.25 \times 10^{-8} \\ 3 & 6.17 \times 10^{-8} & 1.85 \times 10^{-8} \\ 5 & 1.33 \times 10^{-8} & 4.0 \times 10^{-9} \\ & \text { Table II }\end{array}$

Growth in Transverse Angle and Amplitude due to Wakefleld Effects in the $1 \mathrm{GeV}$ IFEL Accelerator

$\begin{array}{lll}b(\mathrm{~mm}) & \Delta \theta(\mathrm{rad}) & \Delta x(\text { meters }) \\ 1 & 2.78 \times 10^{-6} & 8.33 \times 10^{-6} \\ 2 & 3.47 \times 10^{-7} & 1.05 \times 10^{-6} \\ 3 & 1.03 \times 10^{-7} & 3.09 \times 10^{-7} \\ 5 & 2.22 \times 10^{-8} & 6.67 \times 10^{-8}\end{array}$

\section{Summary}

Tables I and II of section V show that wakefield effects are negligible for both the short demonstration accelerator and for the $1 \mathrm{GeV}$ accelerator. We note that an alignment of $.1 \mathrm{~mm}$ (as we have assumed) should be achievable. Furthermore, even modest focussing would reduce the amplitude growth considerably. For example, if one betatron oscillation occurred in the length of the accelerator, the amplitude growth is reduced by a factor of $2 \pi$.

Recalling that the r.m.s. beam radius from the ATF is $\sim 300 \mu m$, we see that 
an amplitude growth of about $3 \%$ for the $1 \mathrm{GeV}$ accelerator with $b=1 \mathrm{~mm}$. We thus conclude that the use of an optical waveguide for the IFEL accelerator is not excluded by transverse wakefield effects and is worthy of further detailed investigation.

\title{
References
}

1. "Proposal for the Design and Construction of an IFEL Accelerator Demonstration Stage and the Design of a $1 \mathrm{GeV}$ High Brightness IFEL Accelerator," E. Courant, C. Pelligrini, J. Sandweiss A. van Steenbergen, J. Marshall, P. Sprangle, I. Bigio, and N. Kurnit. Submitted to the Department of Energy and the Office of Naval Research June,1987.

2. W. Zakowicz, "Low Loss Metallic Waveguide for Transmission of Optical Radiation," J. Appl. Phys. 55 (9), May 1984.

3. P. B. Wilson, "Physics of High Energy Accelerators (Fermilab Summer School, 1981)," Ed. R. A. Carrigan, F. R. Huson, and M. Month, AIP Conference Proceedings No. 87 American Institute of Physics, New York 1982.

4. N. A. Kurnit, Private Communication. See also N. A. Kurnit, "Whispering Mode Waveguide," SPIE vol. 288 Los Alamos Conference on Optics (1981) p.142.

5. A. W. Chao, "Coherent Instabilities of a Bunched Beam," Lectures presented at the second summer school on IIigh Energy Particle Accelerators, Stanford Linear Accelerator Center August 2-13, 1982. SLAC-Pub-2946.

6. L. Palumbo and V. G. Vaccaro, “Wake Fields, Impedances and Green's Function," CERN Accelerator School Advanced Accelerator Physics, The Queen's College, Oxford, England 16-27 September 1985, p. 341.

\section{DISCLAIMER}

\begin{abstract}
This report was prepared as an account of work sponsored by an agency of the United States Government. Neither the United States Government nor any agency thereof, nor any of their employees, makes any warranty, express or implied, or assumes any legal liability or responsibility for the accuracy, completeness, or usefulness of any information, apparatus, product, or process disclosed, or represents that its use would not infringe privatel; uwned rights. Reference herein to any specific commercial product, process, or service by trade name, trademark, manufacturer, or otherwise does not necessarily constitute or imply its endorsement, recommendation, or favoring by the United States Government or any agency thereof. The views and opinions of authors expressed herein do not necessarily state or reflect those of the United States Goverament or any agency thereof.
\end{abstract}

